\title{
SEMIGROUP COMPACTIFICATIONS OF SEMIDIRECT PRODUCTS
}

\author{
BY
}

H. D. JUNGHENN AND B. T. LERNER

\begin{abstract}
Let $S$ and $T$ be semigroups, $S \odot T$ a semidirect product, and $F$ a $C^{*}$-algebra of bounded, complex-valued functions on $S(T)$. Necessary and sufficient conditions are given for the $F$-compactification of $S(T) T$ to be expressible as a semidirect product of compactifications of $S$ and $T$. This result is used to show that the strongly almost periodic compactification of $S \odot T$ is a semidirect product and that, in certain general cases, the analogous statement holds for the almost periodic compactification and the left uniformly continuous compactification of $S(\mathcal{T} T$. Applications are made to wreath products.
\end{abstract}

1. Introduction. Let $S$ and $T$ be semigroups with identities (each denoted by 1) and $\tau: T \times S \rightarrow S$ a mapping with the following properties: For each $t, t^{\prime} \in T$, $s \in S, \tau(t, \cdot)$ is a homomorphism on $S, \tau\left(t, \tau\left(t^{\prime}, s\right)\right)=\tau\left(t t^{\prime}, s\right), \tau(1, \cdot)$ is the identity map and $\tau(t, 1)=1$. If multiplication on $S \times T$ is defined by

$$
(s, t)\left(s^{\prime}, t^{\prime}\right)=\left(s \tau\left(t, s^{\prime}\right), t t^{\prime}\right)
$$

then $S \times T$ becomes a semigroup, denoted by $S(T) T$ and called a semidirect product of $S$ and $T$.

Let $S$ and $T$ have (Hausdorff) topologies relative to which multiplication in $S, T$ and $S(\tau) T$ (with the product topology) is separately continuous (in the terminology of [1], $S, T$ and $S(T) T$ are semitopological semigroups). In a previous paper the first author, generalizing some results of [10] and [14], showed that if $T$ contains a dense subgroup then the almost periodic compactification $(S \odot T)^{A P}$ of $S \odot T$ is a semidirect product $X \subseteq Y$, where $Y=T^{A P}$ and $X$ is a continuous homomorphic image of $S^{A P}$ [11]. In the present paper we consider various generalizations and extensions of this result. For example, we obtain the above characterization of $(S \mathcal{T} T)^{A P}$ under the considerably weaker assumption that $A P(T)=S A P(T)$, and prove that $(S \mathcal{T} T)^{S A P}$ is always a semidirect product (see $\S 2$ for definitions). These results are derived from a general theorem which gives necessary and sufficient conditions for the spectrum of a left $m$-introverted sub- $C^{*}$-algebra $F$ of $C(S \odot T)$ to be (canonically isomorphic to) a semidirect product of spectra of subalgebras of $C(S)$ and $C(T)$ derived from $F$. Examples are provided to illustrate the limitations of the theory, and applications are made to topological wreath products.

Received by the editors May 22, 1979 and, in revised form, April 21, 1980.

AMS (MOS) subject classifications (1970). Primary 22A15, 22A20, 43A60; Secondary 22A25.

Key words and phrases. Semitopological semigroup, semidirect product, compactification, wreath product, minimal ideal, almost periodic, strongly almost periodic. 
The main results of this paper (special cases of which may be found in [15]) are generalizations of work by the first author on compactifications of direct products of semigroups [12] (see also [6] and [3]).

2. Preliminaries. Throughout this section $S$ denotes a semitopological semigroup and $C(S)$ the $C^{*}$-algebra of bounded continuous complex-valued functions on $S$. The operators $R(t)$ and $L(s)$ on $C(S)$ are defined by

$$
R(t) f(s)=f(s t)=L(s) f(t) \quad(s, t \in S) .
$$

If $F$ is a sub- $C^{*}$-algebra of $C(S)$ then $S^{F}$ denotes the spectrum (= space of nonzero continuous complex homomorphisms) of $F$ equipped with the relativized weak* topology, and $\delta: S \rightarrow S^{F}$ the evaluation mapping. A sub- $C^{*}$-algebra $F$ of $C(S)$ will be called admissible if $F$ contains the constant functions, $F$ is translation invariant (i.e., $R(s) F \cup L(s) F \subset F$ for all $s \in S$ ) and $F$ is left $m$-introverted (i.e., the mapping $f_{y}: s \rightarrow y(L(s) f)$ is a member of $F$ for each $f \in F$ and $y \in S^{F}$ ).

Let $w$ be a continuous function from a semitopological semigroup $T$ into $S$, and $w^{*}: C(S) \rightarrow C(T)$ the dual mapping $\left(w^{*} f=f \circ w\right)$. In $\$ 3$ we shall make frequent use of the following fact: if $w$ is a homomorphism and $F$ is an admissible sub- $C^{*}$-algebra of $C(S)$ then $w^{*}(F)$ is an admissible sub- $C^{*}$-algebra of $C(T)$. (That $w^{*}(F)$ is a $C^{*}$-algebra follows, for example, from [17, p. 43]. The other requirements for admissibility are readily verified.)

If $F \subset C(S)$ is admissible then a binary operation $(x, y) \rightarrow x y$ may be defined on $S^{F}$ (specifically, $\left.x y(f)=x\left(f_{y}\right)\right)$ relative to which the pair $(X, u)=\left(S^{F}, \delta\right)$ has the following properties:

(i) $X$ is a compact Hausdorff topological space and a semigroup such that for each $y \in X$ the mapping $x \rightarrow x y: X \rightarrow X$ is continuous;

(ii) $u: S \rightarrow X$ is a continuous homomorphism with range dense in $X$ such that for each $s \in S$ the mapping $x \rightarrow u(s) x: X \rightarrow X$ is continuous; and

(iii) $u^{*} C(X)=F$.

Any pair $(X, u)$ satisfying (i) and (ii) will be called a right topological compactification of $S$, and an $F$-compactification if, in addition, (iii) holds. We shall refer to the pair $\left(S^{F}, \delta\right)$ as the canonical $F$-compactification. $F$-compactifications of $S$ are unique in the following sense: If $(Y, v)$ also satisfies (i)-(iii) then there exists an (algebraic and topological) isomorphism $w$ from $X$ onto $Y$ such that $w u=v$. (For this and related results see, for example, [2], where the general theory of $F$-compactifications of semigroups is developed in detail.)

A simple example of an admissible subalgebra of $C(S)$ is the space $L U C(S)$ of left uniformly continuous functions on $S$. This is the space of all functions $f \in C(S)$ with the property that the mapping $s \rightarrow L(s) f$ is norm continuous. If $S$ is discrete or a compact topological semigroup then obviously $L U C(S)=C(S)$. If $S$ is a topological group then $L U C(S)$ is the space of all functions on $S$ which are uniformly continuous with respect to the right uniformity. (See [2] and [16] for other properties of $L U C(S)$.)

Let $E$ be a Banach space, $B(E)$ the space of bounded linear operators on $E$, and $V$ a subsemigroup of $B(E)$, where the multiplication in $B(E)$ is understood to be 
operator composition. A vector $f \in E$ is almost periodic with respect to $V$ if $V f=\{v f: v \in V\}$ is relatively norm compact in $E$. If $E=C(S)$ then the set of all functions which are almost periodic with respect to $V$ shall be denoted by $A P(S ; V)$. It is well known that $A P(S ; R(S))=A P(S ; L(S))=A P(S ; R(S) L(S))$ and that this space, which we shall henceforth denote by $A P(S)$ (or simply $A P$ ), is an admissible sub- $C^{*}$-algebra of $C(S)$. Furthermore the $A P$-compactification of $S$ is a compact topological semigroup (i.e., jointly continuous multiplication). (See, for example, [1], [2], [4], or [5].)

Following [5] we shall call a finite-dimensional subspace $D$ of $E V$-unitary if $V D \subset D$ and if the closure of $\left.V\right|_{D}$ in $B(D)$ is a compact group $G$ under composition (with identity the identity operator). Haar measure on $G$ may then be used in the standard way to construct an inner product on $D$ relative to which each member of $\left.V\right|_{D}$ is unitary. If $E=C(S)$ then the closed linear span of all $V$-unitary subspaces of $E$ will be denoted by $\operatorname{SAP}(S ; V)$.

If $H$ is a (complex) Hilbert space and $s \rightarrow U(s)$ is a (strongly) continuous unitary representation of $S$ on $H$, then any function of the form $s \rightarrow(U(s) x, y)(x, y \in H)$ is called a coefficient of the representation, where $(\cdot, \cdot)$ denotes the inner product of $H$. The closed linear span in $C(S)$ of the coefficients of all finite-dimensional continuous unitary representations of $S$ is called the space of strongly almost periodic functions on $S$ and is denoted by $S A P(S)$.

The following lemma is well known. Since we have not found a complete proof in the literature we include here a sketch of one. (In the proofs of Lemmas 2.1 and 2.2 below, we shall frequently use without mention the fact that a closed subsemigroup of a compact topological group is a subgroup.)

LeMma 2.1. $S A P(S)$ is an admissible sub-C*-algebra of $A P(S)$.

Proof. Every coefficient of a finite-dimensional unitary representation of $S$ is almost periodic, and left and right translates of a coefficient are coefficients. Also, using tensor products one shows easily that the product of two coefficients is again a coefficient. Therefore $S A P(S)$ is a translation invariant subalgebra of $A P(S)$ and hence also left $m$-introverted. It remains to show that $S A P(S)$ is conjugate closed.

Let $s \rightarrow U(s)$ be a continuous unitary representation of $S$ on a finite-dimensional Hilbert space $H$. We show that $s \rightarrow(y, U(s) x)$ is a coefficient of such a representation $(x, y \in H)$. Let $D$ be the linear space of all such functions, where $x$ varies but $y$ is fixed. Then $D$ is $R(S)$-unitary, and we may choose an inner product $\langle\cdot, \cdot\rangle$ on $D$ relative to which the operators $\left.R(s)\right|_{D}$ are unitary. Since the closure of $U(S)$ in $B(H)$ is a group, there exists a sequence $\left(t_{n}\right)$ in $S$ such that $U\left(t_{n}\right)$ converges in $B(H)$ to the identity. We may take $\left(t_{n}\right)$ so that $\delta\left(t_{n}\right)$ converges on $D$ to a linear functional $\mu$. Choose $g \in D$ such that $\mu(f)=\langle f, g\rangle(f \in D)$. Then any $f \in D$ is a coefficient:

$$
\begin{aligned}
\langle R(s) f, g\rangle & =\lim _{n} R(s) f\left(t_{n}\right)=\lim _{n}\left(y, U\left(t_{n} s\right) x\right) \\
& =(y, U(s) x)=f(s) .
\end{aligned}
$$

Therefore the complex conjugate of a coefficient is again a coefficient, completing the proof. 
As a consequence of Lemma $2.1, S$ has an $S A P$-compactification. It is easily verified that $S^{S A P}$ is in fact a topological group (see [2, p. 118]).

deLeeuw and Glicksberg have shown that if $S$ has an identity, then $\operatorname{SAP}(S)=$ $S A P(S ; R(S))$ [5]. A special case of the following extension of their result will be useful in $\S 3$.

LEMMA 2.2. Let $S$ be a semitopological semigroup with nonempty center $Z$. Then

$$
S A P(S)=S A P(S ; R(S))=S A P(S ; L(S) R(S))=S A P(L(S)) .
$$

Proof. Since every $L(S) R(S)$-unitary subspace of $C(S)$ is obviously both $L(S)$-unitary and $R(S)$-unitary, we have immediately that

$$
S A P(S ; L(S) R(S)) \subset S A P(S ; L(S)) \cap S A P(S ; R(S)) .
$$

To verify the inclusion

$$
S A P(S) \subset S A P(S ; L(S) R(S)),
$$

let $s \rightarrow U(s)$ be any continuous unitary representation of $S$ on a finite-dimensional Hilbert space $H$, and define $\phi: H \times H \rightarrow C(S)$ by $\phi(x, y)(s)=(U(s) x, y)$. If $D$ is the span of $\phi(H \times H)$ then $D$ is finite dimensional, and the identity $L(s) R(t) \phi(x, y)=\phi\left(U(t) x, U(s)^{-1} y\right)$ shows that $D$ is $L(S) R(S)$-invariant. Let $A$ be a member of the closure of $\left.L(S) R(S)\right|_{D}$ in $B(D)$. There exist sequences $\left(s_{n}\right)$ and $\left(t_{n}\right)$ in $S$, and $V, W \in G=$ closure of $U(S)$ in $B(H)$ such that $L\left(s_{n}\right) R\left(t_{n}\right) \rightarrow A$ on $D$ and $U\left(s_{n}\right) \rightarrow V, U\left(t_{n}\right) \rightarrow W$ in $B(H)$. Since $G$ is a group there exist sequences $\left(r_{n}\right),\left(q_{n}\right)$ in $S$ such that $U\left(r_{n}\right) \rightarrow V^{-1}$ and $U\left(q_{n}\right) \rightarrow W^{-1}$. These sequences may be chosen so that $L\left(r_{n}\right) R\left(q_{n}\right)$ converges on $D$ to some operator $B \in B(D)$. Then for any $x, y \in H$,

$$
\begin{aligned}
A B \phi(x, y) & =\lim _{n} L\left(s_{n}\right) R\left(t_{n}\right) L\left(r_{n}\right) R\left(q_{n}\right) \phi(x, y) \\
& =\lim _{n} \phi\left(U\left(t_{n} q_{n}\right) x, U\left(r_{n} s_{n}\right)^{-1} y\right)=\phi(x, y) .
\end{aligned}
$$

Therefore, the closure of $\left.L(S) R(S)\right|_{D}$ in $B(D)$ is a group, $D$ is $L(S) R(S)$-unitary, and (2) follows.

Next we prove that

$$
S A P(S ; R(S)) \subset S A P(S) .
$$

Let $D$ be any $R(S)$-unitary subspace of $C(S)$. Since the closure of $\left.R(Z)\right|_{D}$ in $B(D)$ is a group, there exists a sequence $\left(s_{n}\right)$ in $Z$ such that $\left.R\left(s_{n}\right)\right|_{D}$ converges to the identity operator. Choose $\left(s_{n}\right)$ so that $\delta\left(s_{n}\right)$ converges on $D$ to some linear functional $\mu$. If $\langle\cdot, \cdot\rangle$ is an inner product on $D$ making each $\left.R(s)\right|_{D}$ unitary, and if $g \in D$ satisfies $\mu(f)=\langle f, g\rangle(f \in D)$, then for any $s \in S$,

$$
\begin{aligned}
\langle R(s) f, g\rangle & =\mu(R(s) f)=\lim _{n} R(s) f\left(s_{n}\right)=\lim _{n} f\left(s_{n} s\right) \\
& =\lim _{n} f\left(s s_{n}\right)=\lim _{n} R\left(s_{n}\right) f(s)=f(s) .
\end{aligned}
$$

Therefore, each member of $D$ is a coefficient, establishing (3).

A similar proof, using the conjugate invariance of $S A P(S)$, shows that

$$
S A P(S ; L(S)) \subset S A P(S) .
$$

Therefore, all four spaces are equal. 
Remark. The inclusions (1) and (2) hold if $Z$ is empty but the other inclusions may fail. As an example, take $S$ to be a left zero semigroup. Then

$$
S A P(S ; R(S))=C(S),
$$

but $S A P(S)=S A P(S ; L(S))=S A P(S ; L(S) R(S))=\mathbf{C}$.

Lemma 2.3. Let $S$ be a semigroup, $D$ and $E$ Banach spaces, $\phi: S \rightarrow B(D)$ and $\psi$ : $S \rightarrow B(E)$ representations (or antirepresentations) of $S$, and $w: D \rightarrow E$ a bounded linear operator such that $\psi(s) w=w \phi(s)(s \in S)$. If $D$ is $\phi(S)$-unitary then $w(D)$ is a $\psi(S)$-unitary subspace of $E$.

Proof. The intertwining property of $w$ shows that $w(D)$ is $\psi(S)$-invariant. Let $A$ be in the closure in $B(w(D))$ of $\left.\psi(S)\right|_{w(D)}$ and $\left(s_{n}\right)$ a sequence in $S$ such that $\left.\psi\left(s_{n}\right)\right|_{w(D)} \rightarrow A$ and such that $\phi\left(s_{n}\right)$ converges in $B(D)$. Since $D$ is $\phi(S)$-unitary there exists a sequence $\left(t_{n}\right)$ in $S$ such that $\phi\left(s_{n}\right) \phi\left(t_{n}\right) x \rightarrow x$ for each $x \in D$. Let $\left(t_{n}^{\prime}\right)$ be a subsequence of $\left(t_{n}\right)$ and $C \in B(w(D))$ such that $\left.\psi\left(t_{n}^{\prime}\right)\right|_{w(D)} \rightarrow C$. If $\left(s_{n}^{\prime}\right)$ denotes the corresponding subsequence of $\left(s_{n}\right)$ then for each $x \in D$,

$$
A C w(x)=\lim _{n} \psi\left(s_{n}^{\prime} t_{n}^{\prime}\right) w(x)=\lim _{n} w\left(\phi\left(s_{n}^{\prime} t_{n}^{\prime}\right) x\right)=w(x) .
$$

Similarly, $C A w(x)=w(x)$. Therefore, $w(D)$ is $\psi(S)$-unitary.

In the next section we shall need the following properties of compact topological semigroups (see, for example, [9]): Every compact topological semigroup $S$ contains a minimal ideal $M(S) . M(S)$ is closed and is the union of the minimal right (left) ideals of $S$. The right (left) ideals of $S$ are exactly the sets $e S$ ( $S e$ ), where $e$ is an idempotent of $M(S)$. As a consequence, if $s \in M(S)$ then there exist idempotents $d$ and $e$ in $M(S)$ such that $d s=s e=s$. An idempotent $e$ in $S$ is said to be primitive if $e f=f e=f^{2}=f$ implies $e=f$. A necessary and sufficient condition for an idempotent $e \in S$ to be primitive is that $e \in M(S)$.

3. Compactifications of $S(T)$. Let $S$ and $T$ denote semitopological semigroups with identities and $S(\tau) T$ a semidirect product as defined in $\S 1$. We shall assume that $S(\tau) T$, with the product topology, is also a semitopological semigroup. Simple sufficient conditions for this are that $S$ be a topological semigroup and $\tau$ be separately continuous, or that $T$ be discrete and $\tau(t, \cdot)$ be continuous for each $t \in T$. In this section, we determine the structure of $F$-compactifications of $S(T) T$ for various admissible sub- $C^{*}$-algebras $F$ of $C(S(T) T)$.

We shall denote by $p_{1}: S\left(\mathcal{T} T \rightarrow S\right.$ and $p_{2}: S(\mathcal{T} T \rightarrow T$ the projection mappings and by $q_{1}: S \rightarrow S\left(\mathcal{T} T\right.$ and $q_{2}: T \rightarrow S\left(\mathcal{T} T\right.$ the injection mapping $\left(q_{1}(s)=(s, 1)\right.$, $\left.q_{2}(t)=(1, t)\right)$. Note that $q_{1}$ and $q_{2}$ are homomorphisms and therefore $q_{1}^{*}(F)$ and $q_{2}^{*}(F)$ are admissible whenever $F$ is admissible. $p_{2}$ is also a homomorphism, but in general $p_{1}$ is not, and it is this latter fact which distinguishes the problem of representing $(S \odot T)^{A P}$ as a semidirect product from the corresponding problem for the direct product case.

The following theorem for the special case of a direct product was essentially obtained in [12, Theorem 1]. 
TheOREM 3.1. Let $F$ be an admissible sub-C*-algebra of $C(S \odot T)$. Then statements (a) and (b) are equivalent. Furthermore, if (a) holds then $(X, u)$ is a $q_{1}^{*}(F)$ compactification of $S$ and $(Y, v)$ is a $q_{2}^{*}(F)$-compactification of $T$.

(a) There exist right topological compactifications $(X, u)$ of $S$ and $(Y, v)$ of $T$ and a semidirect product $X \subseteq Y$ such that $(X @ Y, u \times v)$ is an F-compactification of $S(T) T$.

(b) $\left(q_{i} p_{i}\right)^{*} F \subset F(i=1,2)$, and for each $f \in F$ either $f(S, \cdot)$ is relatively norm compact in $C(T)$ or $f(\cdot, T)$ is relatively norm compact in $C(S)$.

Proof. Assume (a) holds and let $f \in F$. Choose $g \in C(X \times Y)$ such that $f=(u \times v)^{*}(g)$ (property (iii), §2), and define $h \in C(X \times Y)$ by $h(x, y)=$ $g(x, v(1))$. Then $\left(q_{1} p_{1}\right)^{*} f=(u \times v)^{*} h \in F$. Also, since $f(S, \cdot) \subset v^{*}(g(X, \cdot))$, $f(S, \cdot)$ is relatively norm compact in $C(T)$. Similarly, $\left(q_{2} p_{2}\right)^{*} f \in F$, and $f(\cdot, T)$ is relatively norm compact in $C(S)$. Therefore (a) implies (b).

Conversely, assume (b). Let $(X, u)$ be the canonical $q_{1}^{*}(F)$-compactification of $S$ and $(Y, v)$ the canonical $q_{2}^{*}(F)$-compactification of $T$ (see $\S 2$ ). If $g \in q_{1}^{*}(F)$ we shall let $\hat{g}$ denote the unique member of $C(X)$ such that $u^{*}(\hat{g})=g$, and similarly for functions in $q_{2}^{*}(F)$.

We show first that $F$ is the closed linear span of all functions $g_{1} \otimes g_{2}\left(g_{i} \in\right.$ $q_{i}^{*}(F)$ ), where $g_{1} \otimes g_{2}(s, t)=g_{1}(s) g_{2}(t)$. Each such function is in $F$, since if $g_{i}=q_{i}^{*}\left(f_{i}\right)$ then $g_{1} \otimes g_{2}=\left[\left(q_{1} p_{1}\right)^{*} f_{1}\right] \cdot\left[\left(q_{2} p_{2}\right)^{*} f_{2}\right]$. Now let $f \in F$, and assume that the norm closure $A$ of $f(S, \cdot)$ is compact (the case $f(\cdot, T)$ relatively compact is similar). For each $s \in S, f(s, \cdot)=q_{2}^{*} L(s, 1) f$, hence $A \subset q_{2}^{*}(F)$. Also, for each $t \in T, f(\cdot, t)=q_{1}^{*} R(1, t) f \in q_{1}^{*}(F)$, hence we may define a map $\tilde{f}$ from $X$ into the bounded functions on $T$ by $\tilde{f}(x)(t)=x(f(\cdot, t))(x \in X, t \in T)$. Let $x \in X$ and $\left(u\left(s_{n}\right)\right)$ a net converging to $x$. Then $f(x)(t)=\lim _{n} f\left(s_{n}, t\right)$, and since $f(S, \cdot)$ is relatively norm compact, this convergence must be uniform in $t \in T$. Therefore $\tilde{f}(X) \subset A$, and it follows easily that $\tilde{f}: X \rightarrow q_{2}^{*}(F)$ is continuous with respect to the weak* topology on $X$ and the norm topology on $q_{2}^{*}(F)$. Thus $\tilde{f}$ is the uniform limit in $x \in X$ and $t \in T$ of finite sums of functions $\hat{g}_{1} \otimes g_{2}, \hat{g}_{1} \in C(X), g_{2} \in q_{2}^{*}(F)$ (see, for example, [18, p. 448]). Since $u^{*} C(X)=q_{1}^{*}(F), f$ has the desired approximation property.

By the Stone-Weierstrass Theorem, the functions $\hat{g}_{1} \otimes \hat{g}_{2}\left(g_{i} \in q_{i}^{*}(F)\right)$ span a dense subspace of $C(X \times Y)$. Since $(u \times v)^{*}\left(\hat{g}_{1} \otimes \hat{g}_{2}\right)=g_{1} \otimes g_{2}$, it follows from the result of the preceding paragraph that $(u \times v)^{*}$ maps $C(X \times Y)$ onto $F$. Therefore, if $(Z, w)$ is an $F$-compactification of $S(T),\left(w^{*}\right)^{-1}(u \times v)^{*}$ maps $C(X \times Y)$ isomorphically and isometrically onto $C(Z)$ and is thus the dual of a homeomorphism $\phi: Z \rightarrow X \times Y$ such that $u \times v=\phi w$.

Now give $X \times Y$ the unique multiplication which makes $\phi$ a semigroup isomorphism. Properties (i)-(iii) of $\S 2$ are easily seen to hold for $(X \times Y, u \times v)$, and it remains to construct a mapping $\rho: Y \times X \rightarrow X$ with the appropriate properties such that $X \times Y$ is the semidirect product $X @ Y$. Let $\pi: X \times Y \rightarrow X$ denote the projection mapping and define $\rho(y, x)=\pi[(u(1), y)(x, v(1))]$. Note that $\rho(\cdot, x)$ and 
$\rho(v(t), \cdot)$ are continuous for each $x \in X, t \in T$, and that

$$
\begin{aligned}
\rho(v(t), u(s)) & =\pi[(u(1), v(t))(u(s), v(1))] \\
& =\pi[(u \times v)(1, t)(u \times v)(s, 1)] \\
& =\pi[(u \times v)(\tau(t, s), t)]=u(\tau(t, s)) .
\end{aligned}
$$

In particular $\rho(v(1), \cdot)$ is the identity mapping and $\rho(y, u(1))=u(1)$. For any $s$, $s^{\prime} \in S$ and $t, t^{\prime} \in T$,

$$
\begin{aligned}
(u(s), v(t))\left(u\left(s^{\prime}\right), v\left(t^{\prime}\right)\right) & =(u \times v)\left(s \tau\left(t, s^{\prime}\right), t t^{\prime}\right)=\left(u(s) u\left(\tau\left(t, s^{\prime}\right)\right), v(t) v\left(t^{\prime}\right)\right) \\
& =\left(u(s) \rho\left(v(t), u\left(s^{\prime}\right)\right), v(t) v\left(t^{\prime}\right)\right) .
\end{aligned}
$$

Letting successively $u\left(s^{\prime}\right) \rightarrow x^{\prime}, v\left(t^{\prime}\right) \rightarrow y^{\prime}, v(t) \rightarrow y$ and $u(s) \rightarrow x$ gives $(x, y)\left(x^{\prime}, y^{\prime}\right)$ $=\left(x \rho\left(y, x^{\prime}\right), y y^{\prime}\right)$. From this we have

$$
\begin{aligned}
\left(\rho(y, x) \rho\left(y, x^{\prime}\right), y\right) & =(\rho(y, x), y)\left(x^{\prime}, v(1)\right)=(u(1), y)(x, v(1))\left(x^{\prime}, v(1)\right) \\
& =(u(1), y)\left(x x^{\prime}, v(1)\right)=\left(\rho\left(y, x x^{\prime}\right), y\right),
\end{aligned}
$$

so $\rho(y, \cdot)$ is a homomorphism. Also,

$$
\begin{aligned}
\left(\rho\left(y y^{\prime}, x\right), y y^{\prime}\right) & =\left(u(1), y y^{\prime}\right)(x, v(1))=(u(1), y)\left(u(1), y^{\prime}\right)(x, v(1)) \\
& =(u(1), y)\left(\rho\left(y^{\prime}, x\right), y^{\prime}\right)=\left(\rho\left(y, \rho\left(y^{\prime}, x\right)\right), y y^{\prime}\right),
\end{aligned}
$$

hence $\rho\left(y y^{\prime}, x\right)=\rho\left(y, \rho\left(y^{\prime}, x\right)\right)$. Thus, $\rho$ has the required properties and $X \times Y$ is the semidirect product $X \subseteq Y$. Therefore (b) implies (a).

Finally, if (a) holds then $(u \times v)^{*} C(X \times Y)=F$, from which it follows easily that $u^{*} C(X)=q_{1}^{*}(F)$ and $v^{*} C(Y)=q_{2}^{*}(F)$.

Our first application of Theorem 3.1 is to the almost periodic compactification of $S(\mathcal{T} T$. Before stating the result we introduce the following notation: Define $\psi$ : $S(\tau) T \rightarrow B(C(S))$ by $\psi(s, t) f\left(s^{\prime}\right)=f\left(s \tau\left(t, s^{\prime}\right)\right)$. Then $\psi$ is an antirepresentation of $S\left(\mathcal{T} T\right.$, and $A P(S ; \psi(S \odot T))$ is a left translation invariant sub- $C^{*}$-algebra of $A P(S)$.

THEOREM 3.2. Statements (a), (b) and (c) of the following list are equivalent and are implied by any one of the conditions (d) to (j). Furthermore, if (a) holds then $(Y, v)$ is an AP-compactification of $T, A P(S ; \psi(S \odot T))$ is admissible, and $(X, u)$ is an $A P(S ; \psi(S \mathcal{T} T))$-compactification of $S$.

(a) There exist right topological compactifications $(X, u)$ of $S$ and $(Y, v)$ of $T$ and $a$ semidirect product $X \subseteq Y$ such that $(X @ Y, u \times v)$ is an AP-compactification of $S(\tau) T$.

(b) $q_{1}^{*} A P(S \odot T) \subset A P(S ; \psi(S \odot T))$.

(c) $\left(q_{1} p_{1}\right)^{*} A P(S \odot T) \subset A P(S \odot T)$.

(d) $A P(T)=S A P(T)$.

(e) For each $t \in T$, either $R(t)$ or $L(t)$ (as operators on $A P(T)$ ) is an isometry.

(f) There exists a dense subset $Q$ of $T$ such that for each $t \in Q$, either $T t$ or $t T$ is dense in $T$.

(g) $T$ is topologically left or right simple.

(h) $T$ contains a dense subgroup.

(i) $T$ is compact and the set of mappings $\tau(\cdot, S)$ is equicontinuous.

(j) $S$ is compact and the set of mappings $\tau(T, \cdot)$ is equicontinuous. 
Proof. Let $F=A P(S \mathcal{T} T), H=A P(S ; \psi(S \mathcal{T} T))$. One easily checks that the compactness condition of Theorem $3.1(\mathrm{~b})$ is satisfied. The identities $L(t) q_{2}^{*}=$ $q_{2}^{*} L(1, t)$ and $L(s, t) p_{2}^{*}=p_{2}^{*} L(t)$ show, respectively, that $q_{2}^{*} F \subset A P(T)$ and $p_{2}^{*} A P(T) \subset F$. Therefore $\left(q_{2} p_{2}\right)^{*} F \subset F$ and $q_{2}^{*} F=A P(T)$. The identity

$$
L(s, t) p_{1}^{*}=p_{1}^{*} \psi(s, t)
$$

shows that $p_{1}^{*} H \subset F$ and hence $H \subset q_{1}^{*} F$. Therefore if (b) holds, $H=q_{1}^{*} F$ and $\left(q_{1} p_{1}\right)^{*} F=p_{1}^{*} H \subset F$, and hence (a) follows from Theorem 3.1. The same theorem shows that (a) implies (c). That (c) implies (b) follows immediately from the identity

$$
\psi(s, t) q_{1}^{*}=q_{1}^{*} L(s, t)\left(q_{1} p_{1}\right)^{*} .
$$

Now assume that (d) holds. Let $V$ denote the strong operator closure of $R(1 \times T)$ in $B(F)$. Then $V$ is a compact topological semigroup containing a dense continuous image of $T$ [5, Theorem 3.2], hence, there exists a continuous homomorphism from $T^{A P}$ onto $V$, as may be directly verified. (It may be shown that $V$ is an $A P$-compactification of $T$, but we shall not need this fact here.) Since $A P(T)=$ $S A P(T), T^{A P}$ is a group and therefore so is $V$. Thus each member of $R(1 \times T)$ is invertible in $B(F)$. The identity $\psi(s, t) q_{1}^{*} f=q_{1}^{*} L(s, t) R(1, t)^{-1} f(f \in F)$ then shows that (b) is satisfied.

Next assume that (e) holds. Condition (d) will follow if we show that $T^{A P}$ is a group [2, III.10.4]. For this we use the structure theory of compact topological semigroups (see $\S 2$ ). Denote $T^{A P}$ by $Y$. Let $e$ be any idempotent in $M(Y)$ and $\left(t_{n}\right)$ a net in $T$ such that $\delta\left(t_{n}\right) \rightarrow e$. Since $R(T)$ and $L(T)$ are relatively compact in the strong operator topology of $B(A P(T))$ [5, Theorem 3.2], $\left(t_{n}\right)$ may be chosen so that either $\left(R\left(t_{n}\right)\right)$ or $\left(L\left(t_{n}\right)\right)$ is a net of isometries converging in that topology to some operator $U$. Assume the former (the other case is similar). Then for each $h \in$ $A P(T)$ and $t \in T$,

$$
U h(t)=\lim _{n} R\left(t_{n}\right) h(t)=\lim _{n} L(t) h\left(t_{n}\right)=e(L(t) h),
$$

and similarly, $U^{2} h(t)=e^{2}(L(t) h)=e(L(t) h)$. Therefore $U^{2}=U$, and since $U$ is an isometry, $U$ must be the identity operator. In particular, $h(1)=U h(1)=$ $e(L(1) h)=e(h)(h \in A P(T))$, so $\delta(1)=e$. Thus $\delta(1)$ is the only idempotent in $M(Y)$, so $M(Y)$ is a group and $M(Y)=Y$.

Clearly (f) implies (e) and is implied by either (g) or (h). If (i) holds and if $f \in F$, then the identity $\psi(s, t) q_{1}^{*} f=\psi(1, t) q_{1}^{*} L(s, 1) f$ shows that $q_{1}^{*} f \in H$. Therefore, (i) implies (b).

Finally, we show that (j) implies (c). Let $f \in F, g=\left(q_{1} p_{1}\right)^{*} f$, and $\left(\left(s_{n}, t_{n}\right)\right)$ any net in $S \odot T$. We may assume that $\left(s_{n}\right)$ converges in $S$ to, say, $s^{\prime}$. We shall show that $R\left(s_{n}, t_{n}\right) g(s, t)=f\left(s \tau\left(t, s_{n}\right), 1\right)$ converges uniformly in $(s, t)$ to $f\left(s \tau\left(t, s^{\prime}\right), 1\right)$. If not, then there exists $\varepsilon>0$ and a subnet $\left(s_{k}^{\prime}=s_{n(k)}\right)$ of $\left(s_{n}\right)$ and nets $\left(s_{k}\right),\left(t_{k}\right)$ such that for all $k$,

$$
\left|f\left(s_{k} \tau\left(t_{k}, s_{k}^{\prime}\right), 1\right)-f\left(s_{k} \tau\left(t_{k}, s^{\prime}\right), 1\right)\right| \geqslant \varepsilon .
$$

We may assume that $L\left(s_{k}, 1\right) f$ converges in norm to some $h \in F$. In particular,

$$
f\left(s_{k} \tau\left(t_{k}, s_{k}^{\prime}\right), 1\right)-h\left(\tau\left(t_{k}, s_{k}^{\prime}\right), 1\right) \rightarrow 0
$$


and

$$
f\left(s_{k} \tau\left(t_{k}, s^{\prime}\right), 1\right)-h\left(\tau\left(t_{k}, s^{\prime}\right), 1\right) \rightarrow 0 .
$$

This and the equicontinuity of $\tau(T, \cdot)$ imply that

$$
f\left(s_{k} \tau\left(t_{k}, s_{k}^{\prime}\right), 1\right)-f\left(s_{k} \tau\left(t_{k}, s^{\prime}\right), 1\right) \rightarrow 0,
$$

which contradicts the above inequality. Therefore $g \in F$ completing proof.

REMARKs. The implication (h) $\Rightarrow$ (a) was obtained (by different methods) in [11].

If $S(\tau) T$ is a direct product $(\tau(t, s)=s$ for all $s$ and $t)$, then $A P(S: \psi(S(\mathcal{T} T))=$ $A P(S)$ and hence (b) automatically holds. Thus Theorem 3.2 includes the result of deLeeuw and Glicksberg on $A P$-compactifications of direct products [6]. (See also [3], [12].)

The $A P$-compactification of $S(T) T$ cannot always be represented as a semidirect product, as the following example demonstrates: Let $S$ be the interval $[a, 1]$ $(0<a<1)$ with the usual topology and with multiplication $s * s^{\prime}=s s^{\prime} \vee a$ (where $s s^{\prime}$ denotes the ordinary product of $s$ and $s^{\prime}$ ), and let $T$ be the set of positive integers with the discrete topology and the usual multiplication. Define $\tau: T \times S$ $\rightarrow S$ by $\tau(n, s)=s^{n} \vee a$. The function $f(s, n)=s^{1 / n}$ is in $A P(S \mathcal{T} T)$ as is easily seen from the identity $L(s, n) f(r, m)=s^{1 / m n} f(r, m) \vee a^{1 / n m}$. On the other hand, the identity $\psi(1, n) q_{1}^{*} f(r)=r^{n} \vee a$ shows that $q_{1}^{*} f \notin A P(S ; \psi(S \odot T))$. Therefore, condition (b) of Theorem 3.2 does not hold.

Our next result gives sufficient conditions for the minimal ideal of $(S \mathcal{T} T)^{A P}$ to decompose into a semidirect product.

TheOREM 3.3. If (a) of Theorem 3.2 holds, and if the minimal ideal of $X$ satisfies $M(X) \subset \rho(y, X)$ for each $y \in M(Y)$,

then

$$
M(X @ Y)=M(X) @ M(Y) .
$$

Condition (6) is satisfied if (d) of Theorem 3.2 holds or if $\tau(t, S)$ is dense in $S$ for each $t$ in a dense subset of $T$.

Proof. For any $y \in M(Y), I=\{x \in X: \rho(y, x) \in M(X)\}$ is nonempty by (6) and is an ideal of $X$, so $M(X) \subset I$ and therefore $\rho(y, M(X)) \subset M(X)$. It follows that $M(X) \subseteq M(Y)$ is defined.

Next, let us show that

$$
\rho(y, M(X))=M(X) \quad(y \in M(Y)),
$$

and

$$
\rho(e, c)=c \quad\left(e^{2}=e \in M(Y), c \in M(X)\right) .
$$

To verify (8) it is enough to show that $\rho(y, M(X))$ is an ideal of $X$. Let $x \in X$, $a \in M(X)$ and let $d$ be any member of $M(X)$ such that $d \rho(y, a)=\rho(y, a)$. By (6) there exists $b \in X$ such that $x d=\rho(y, b)$. Then

$$
x \rho(y, a)=x d \rho(y, a)=\rho(y, b) \rho(y, a)=\rho(y, b a),
$$

which shows that $\rho(y, M(X))$ is a left ideal of $X$. A similar argument shows that it is also a right ideal. This establishes (8). For (9), given $e$ and $c$ choose $x \in X$ such that $\rho(e, x)=c$. Then $c=\rho\left(e^{2}, x\right)=\rho(e, \rho(e, x))=\rho(e, c)$. 
From (8) we conclude that $\rho(y, M(X))=M(X)$ for all $y \in Y$. Indeed, if $y \in Y$ and $y^{\prime} \in M(Y)$ then by (8), $\rho(y, M(X))=\rho\left(y, \rho\left(y^{\prime}, M(X)\right)\right)=\rho\left(y y^{\prime}, M(X)\right)=$ $M(X)$. It follows easily that $M(X) @ M(Y)$ is an ideal of $X \subseteq Y$ and hence that $M(X @ Y) \subset M(X) @ M(Y)$.

Now let $(a, b) \in M(X) @ M(Y)$. Assume first that $a$ and $b$ are idempotents. By (9), $(a, b)$ is an idempotent, hence $(a, b)$ will be in $M(X \odot Y)$ if we show that it is primitive. Let $(x, y) \in X @ Y$ such that $(x, y)^{2}=(x, y)=(a, b)(x, y)=$ $(x, y)(a, b)$. Then $x \rho(y, x)=x=a \rho(b, x)=x \rho(y, a)$, and $y^{2}=y=b y=y b$. Since $b \in M(Y), b$ is primitive so $y=b$. Also by (9), $\rho(b, a)=a$ and $\rho(b, x)=x$. Therefore $x^{2}=x=a x=x a$, and since $a$ is primitive, $x=a$.

In the general case, choose idempotents $d \in M(X), e \in M(Y)$ such that $a d=a$ and $b e=b$. By (8) the compact subsemigroup $\{x \in M(X): \rho(b, x)=d\}$ is nonempty and therefore contains an idempotent $f$. Then $(a, b)(f, e)=(a \rho(b, f), b e)=$ $(a d, b e)=(a, b)$. By the result of the preceding paragraph $(f, e) \in M(X @ Y)$, hence $(a, b) \in M(X @ Y)$. This establishes (7).

If (d) of Theorem 3.2 holds, then $Y$ is a group and hence (6) is obviously satisfied. If $\tau(t, S)$ is dense in $S$ then $\rho(v(t), u(S))=u(\tau(t, S))$ is dense in $u(S)$, and therefore $\rho(v(t), X)=X$. If this holds for each $t$ in a dense subset of $T$ then the joint continuity of $\rho$ implies that $\rho(y, X)=X$ for all $y \in Y$.

REMARKS. If 3.2(d) holds then the minimal right ideals of $X \subseteq Y$ are precisely the sets $I \times Y$, where $I$ is a minimal right ideal of $X$.

If condition (6) does not hold then $M(X) \subseteq M(Y)$ may not even be defined. As an example, let $X$ be a compact topological semigroup with identity, $Y$ the set of integers $\{0,1\}$ with ordinary multiplication, and let $\rho$ be defined by $\rho(0, x)=1$, $\rho(1, x)=x$. Then $M(X @ Y)=X @\{0\}=X @ M(Y)$, hence $M(X) @ M(Y)$ is defined and equals $M(X \odot Y)$ if and only if $M(X)=X$.

Condition (6), however, is not necessary for (7) to hold. For example, let $X=Y=\left\{1, x_{1}, x_{2}\right\}$ with the discrete topology, where 1 is the identity and $x_{i} x_{j}=$ $x_{i}$ for any $i, j$. Define $\rho$ by $\rho(1, x)=x, \rho\left(x_{i}, x_{j}\right)=x_{i}$ and $\rho\left(x_{i}, 1\right)=1$. Then $M(X)=\left\{x_{1}, x_{2}\right\}, \rho\left(x_{1}, X\right)=\left\{1, x_{1}\right\}$, but $M(X @ Y)=M(X) @ M(Y)$. (The authors wish to thank the referee for suggesting this example.)

Our next result asserts that, in contrast to the almost periodic case, the $S A P$ compactification of $S \odot T$ is always a semidirect product. As with Theorem 3.2, it is convenient to state the result in terms of the mapping $\psi$ defined earlier.

THEOREM 3.4. $S A P(S ; \psi(S \mathcal{T} T))$ is an admissible sub-C*-algebra of $S A P(S)$, and if $(X, u)$ is an $\operatorname{SAP}(S ; \psi(S \odot T))$-compactification of $S$ and $(Y, v)$ an SAP-compactification of $T$, then there exists a semidirect product $X \subseteq Y$ such that $(X \subseteq Y$, $u \times v)$ is an $S A P$-compactification of $S(T) T$.

Proof. Let $F=S A P(S \odot T), H=S A P(S ; \psi(S \odot T))$. Since $q_{2}$ and $p_{2}$ are continuous homomorphisms, it follows directly from the definition of $S A P$ that $q_{2}^{*} F \subset S A P(T)$ and $p_{2}^{*} S A P(T) \subset F$. Therefore, $q_{2}^{*} F=S A P(T)$ and $\left(q_{2} p_{2}\right)^{*} F \subset$ $F$. Also, the identity (5) together with Lemmas 2.2 and 2.3 imply that $p_{1}^{*} H \subset F$ and 
hence $H \subset q_{1}^{*} F$. (Note that Lemma 2.2 is applicable here since $S, T$, and, hence, $S(\tau) T$, have identities.) The desired conclusion will follow from Theorem 3.1 once we show that $q_{1}^{*} F \subset H$.

Let $D$ be an $R(S \mathcal{T} T) L(S \mathcal{T} T)$-unitary subspace of $C(S \mathcal{T} T)$. Then $R(1, t)$ (as an operator on $D)$ is invertible in $B(D)$ so we may define $\phi(s, t)=L(s, t) R(1, t)^{-1}$. Obviously, $\phi$ is an antirepresentation of $S \odot T$ on $D$, and $D$ is $\phi(S \mathcal{T} T)$-unitary. A simple calculation shows that $\psi(s, t) q_{1}^{*}=q_{1}^{*} \phi(s, t)$ on $D$. By Lemma 2.3 , then, $q_{1}^{*}(D)$ is $\psi(S \mathcal{C} T)$-unitary. Therefore $q_{1}^{*}(D) \subset H$, and the inclusion $q_{1}^{*}(F) \subset H$ follows from Lemma 2.2 .

We have been unable, so far, to find a satisfactory application of Theorem 3.1 to the space of weakly almost periodic functions (see [1], [2], [4], [5], or [8] for definition). This is hardly surprising since even in the case of direct products stringent conditions must be imposed for a $W A P$-analog of 3.2(a) to hold [3], [12]. The $L U C$ case is somewhat more amenable to treatment, and as an example we offer the following result, the proof of which is a straightforward application of Theorem 3.1 to $\operatorname{LUC}(S \mathcal{T} T)$.

THEOREM 3.5. Let $S$ be a compact topological semigroup and suppose that $\tau$ : $T \times S \rightarrow S$ is jointly continuous. If $u: S \rightarrow S$ denotes the identity mapping and $(Y, v)$ is an LUC-compactification of $T$, then there exists a semidirect product $S @ Y$ such that $(S @ Y, u \times v)$ is an LUC-compactification of $S(T) T$.

4. Application to wreath products. Let $T$ be a discrete semigroup with identity acting on the right on a set $\Lambda$. Thus, there exists a mapping $(\alpha, t) \rightarrow \alpha t: \Lambda \times T \rightarrow$ $\Lambda$ such that $(\alpha t) t^{\prime}=\alpha\left(t t^{\prime}\right)$ and $\alpha 1=\alpha$. (The standard example is $\Lambda=T$ with right multiplication as the action.) Let $S$ be a semitopological semigroup with identity and $S^{\Lambda}$ the product space with the usual coordinate multiplication and product topology. A typical point of $S^{\Lambda}$ will be denoted by $a=\left(s_{\alpha}\right)$. Define $\tau: T \times S^{\Lambda} \rightarrow$ $S^{\Lambda}$ by $\tau(t, a)(\alpha)=a(\alpha t)\left(a \in S^{\Lambda}, t \in T, \alpha \in \Lambda\right)$. The semidirect product $S^{\Lambda}(\tau) T$ is called an (abstract) topological wreath product of $S$ and $T$ and is denoted by $S$ wr $T$. (A survey of the algebraic theory of wreath products of semigroups may be found in [19]. For topological questions, see, for example, [7], [13].)

The following theorems illustrate how the material in $\$ 3$ may be applied to gain information about the structure of certain $F$-compactifications of $S$ wr $T$. A theorem on general $F$-compactifications of $S$ wr $T$ may be obtained by combining Theorem 3.1 of the previous section with Theorem 3 of [12], but we shall restrict ourselves here to the cases $F=A P(S$ wr $T)$ and $F=S A P(S$ wr $T)$.

TheOREM 4.1. Let $(Z, w)$ be an AP-compactification of $S$ and $(Y, v)$ an AP-compactification of $T$. If $T$ is left or right simple then there exists a semidirect product $X @ Y$ such that $(X @ Y, u \times v)$ is an AP-compactification of $S$ wr $T$, where $X$ is the image of the compact topological semigroup $Z^{\Lambda}$ under a continuous homomorphism $\theta$, and $u: S^{\Lambda} \rightarrow X$ is defined by $u\left(\left(s_{\alpha}\right)\right)=\theta\left(\left(w\left(s_{\alpha}\right)\right)\right)$. Furthermore, the minimal ideal of $(S \text { wr } T)^{A P}$ may be expressed as $M(X) \bigodot Y$, and $M(X)=\theta\left(M(Z)^{\Lambda}\right)$.

Proof. By Theorem 3.2, $H=A P\left(S^{\Lambda} ; \psi\left(S^{\Lambda} \odot T\right)\right)$ is admissible, and if $(X, u)$ is an $H$-compactification of $S^{\Lambda}$, then there exists a semidirect product $X \subseteq Y$ such 
that $(X \subseteq Y, u \times v)$ is an $A P$-compactification of $S$ wr $T$. By Corollary $1, \S 4$ of [12], $\left(Z^{\Lambda}, w^{\prime}\right)$ is an $A P$-compactification of $S^{\Lambda}$, where $w^{\prime}: S^{\Lambda} \rightarrow Z^{\Lambda}$ is defined by $w^{\prime}\left(\left(s_{\alpha}\right)\right)=\left(w\left(s_{\alpha}\right)\right)$. Since $H \subset A P\left(S^{\Lambda}\right)$, there exists a continuous homomorphism $\theta$ of $Z^{\Lambda}$ onto $X$ such that $\theta w^{\prime}=u$ [5, Theorem 6.2]. This proves the first part of the theorem. The second part follows from Theorem 3.3 and the fact that $M\left(Z^{\Lambda}\right)=$ $M(Z)^{\Lambda}$.

A similar argument, using Theorem 3.4 of the previous section, Corollary $1, \S 4$ of [12], and the universal mapping property of $S A P$-compactifications $[2, \mathrm{p} .118]$ may be used to establish the following result:

THEOREM 4.2. Let $(Z, w)$ be an SAP-compactification of $S$ and $(Y, v)$ an $S A P$ compactification of $T$. Then there exists a semidirect product $X \subseteq Y$ such that $(X \subseteq Y, u \times v)$ is an $S A P$-compactification of $S$ wr $T$, where $X$ is the image of the compact topological group $Z^{\Lambda}$ under a continuous homomorphism $\theta$, and $u: S^{\Lambda} \rightarrow X$ is defined by $u\left(\left(s_{\alpha}\right)\right)=\theta\left(\left(w\left(s_{\alpha}\right)\right)\right)$.

The authors wish to thank the referee for suggesting several improvements to the exposition of the paper.

\section{REFERENCES}

1. J. F. Berglund and K. H. Hofmann, Compact semitopological semigroups and weakly almost periodic functions, Lecture Notes in Math., vol. 42, Springer-Verlag, Berlin, 1967.

2. J. F. Berglund, H. D. Junghenn and P. Milnes, Compact right topological semigroups and generalizations of almost periodicity, Lecture Notes in Math., vol. 663, Springer-Verlag, Berlin, 1978.

3. J. F. Berglund and P. Milnes, Algebras of functions on semitopological left groups, Trans. Amer. Math. Soc. 222 (1976), 157-178.

4. R. B. Burckel, Weakly almost periodic functions on semigroups, Gordon \& Breach, New York, 1970.

5. K. deLeeuw and I. Glicksberg, Applications of almost periodic compactifications, Acta Math. 105 (1961), 63-97.

6. __ Almost periodic functions on semigroups, Acta Math. 105 (1961), 99-140.

7. J. R. Durbin, On locally compact wreath products, Pacific J. Math. 57 (1975), 99-107.

8. W. F. Eberlein, Abstract ergodic theorems and weak almost periodic functions, Trans. Amer. Math. Soc. 67 (1949), 217-240. 1966.

9. K. H. Hofmann and P. S. Mostert, Elements of compact semigroups, Merrill, Columbus, Ohio,

10. H. D. Junghenn, Almost periodic compactifications of transformation semigroups, Pacific J. Math. 57 (1975), 207-216.

11. Almost periodic functions on semidirect products of transformation semigroups, Pacific $\mathrm{J}$. Math. 79 (1978), 117-128.

12. $\ldots, C^{*}$-algebras of functions on direct products of semigroups, Rocky Mountain J. Math. 10 (1980), 589-597.

13. A. Lakshmi, Topological wreath products, J. Austral. Math. Soc. 5 (1965), 396-400.

14. M. Landstad, On the Bohr compactification of a transformation groüp, Math. Z. 127 (1972), 167-178.

15. B. T. Lerner, $C^{*}$-algebra compactifications of semidirect products of semitopological semigroups, Thesis, George Washington Univ., Washington, D. C., 1979.

16. I. Namioka, On certain actions of semigroups on L-spaces, Studia Math. 29 (1967), 63-77.

17. S. Sakai, $C^{*}$-algebras and $W^{*}$-algebras, Ergebnisse der Mathematik und ihrer Grenzgebiete, Band 60, Springer-Verlag, Berlin, 1971.

18. F. Treves, Topological vector spaces, distributions and kernels, Academic Press, New York, 1967.

19. C. Wells, Some applications of the wreath product construction, Amer. Math. Monthly 83 (1976), 317-338.

Department of Mathematics, The George Washington University, Washington, D. C. 20052

Department of Mathematics, U. S. Naval Academy, Annapolis, Maryland 21402 